\title{
A large symptomatic jejunal subepithelial tumor treated with endoloop-assisted polypectomy using spiral
} enteroscopy

\author{
Jen-Wei Chou ${ }^{1,3,4}$, Ken-Sheng Cheng ${ }^{1}$, Ching-Pin Lin ${ }^{2,3,4}$ \\ ${ }^{1}$ Division of Gastroenterology and Hepatology, Department of Internal Medicine, China Medical University Hospital, College \\ of Medicine, China Medical University, Taichung, Taiwan, ROC, ${ }^{2}$ Division of Gastroenterology, Department of Internal Medicine, \\ Chung Shan Medical University Hospital, Taichung, Taiwan, ROC, ${ }^{3}$ Taiwan Society of Inflammatory Bowel Disease, ${ }^{4}$ Taiwan \\ Association for the Study of Small Intestinal Diseases
}
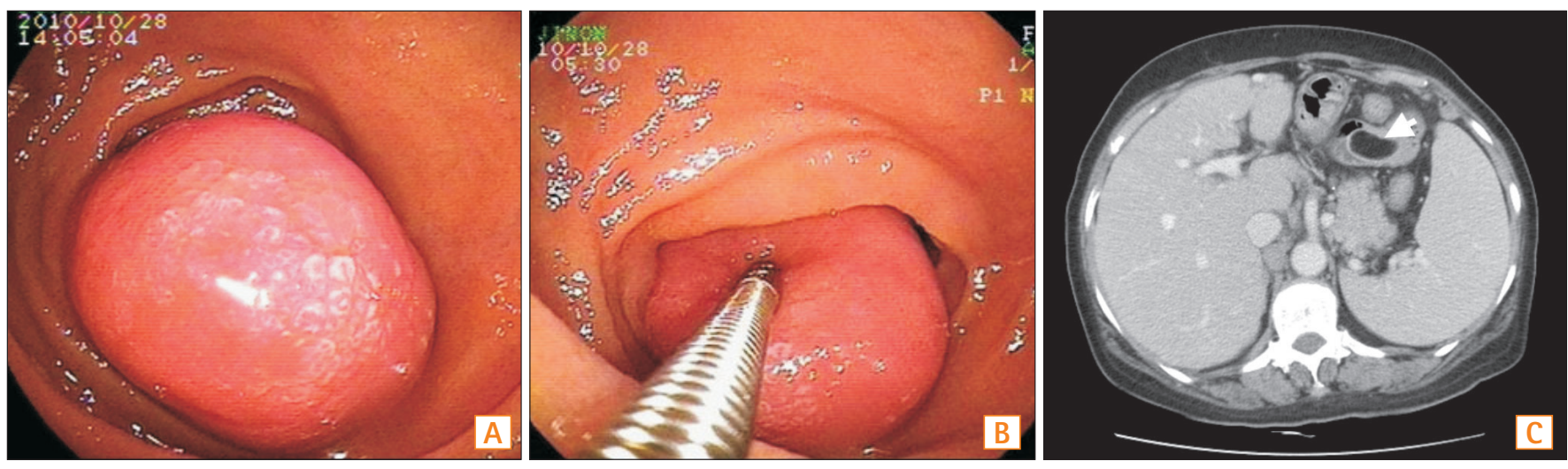

Question: A 70-year-old woman presented to our hospital because of a 6-month history of melena. She had had a medical history of chronic hepatitis B and hepatitis $\mathrm{C}$ for decades. Moreover, she underwent a laparotomy 20 years previously. Physical examination revealed unremarkable abnormalities. Laboratory tests revealed a hemoglobin level of $9 \mathrm{~g} / \mathrm{dL}$. Esophagogastroduodenoscopy, colonoscopy, and small bowel series all failed to detect a bleeding source. Subsequently, spiral enteroscopy was performed perorally by using a specialized overtube (Endo-Ease Discovery Small Bowel; Spirus Medical, Inc., Stoughton, MA, USA) and an enteroscope (Fujinon EN-450T5; Fujinon Corp., Saitama, Japan). A giant subepithelial tumor with superficial ulceration, measuring 3-4 cm in diameter, was identified in the proximal jejunum (Fig. A). Spiral enteroscopy revealed a thickstalk tumor with a "cushion sign" by a grasping forceps (Fig. B). A gastrointestinal stromal tumor was suspected initially; however, the tumor revealed fat content with an attenuation coefficient of -98 Hounsfield units on CT (Fig. C. arrow). We decided to remove it endoscopically to prevent further risk of rebleeding or obstruction and applied an endoloop to ligate the stalk of the tumor (Fig. D). After looping of the tu- 

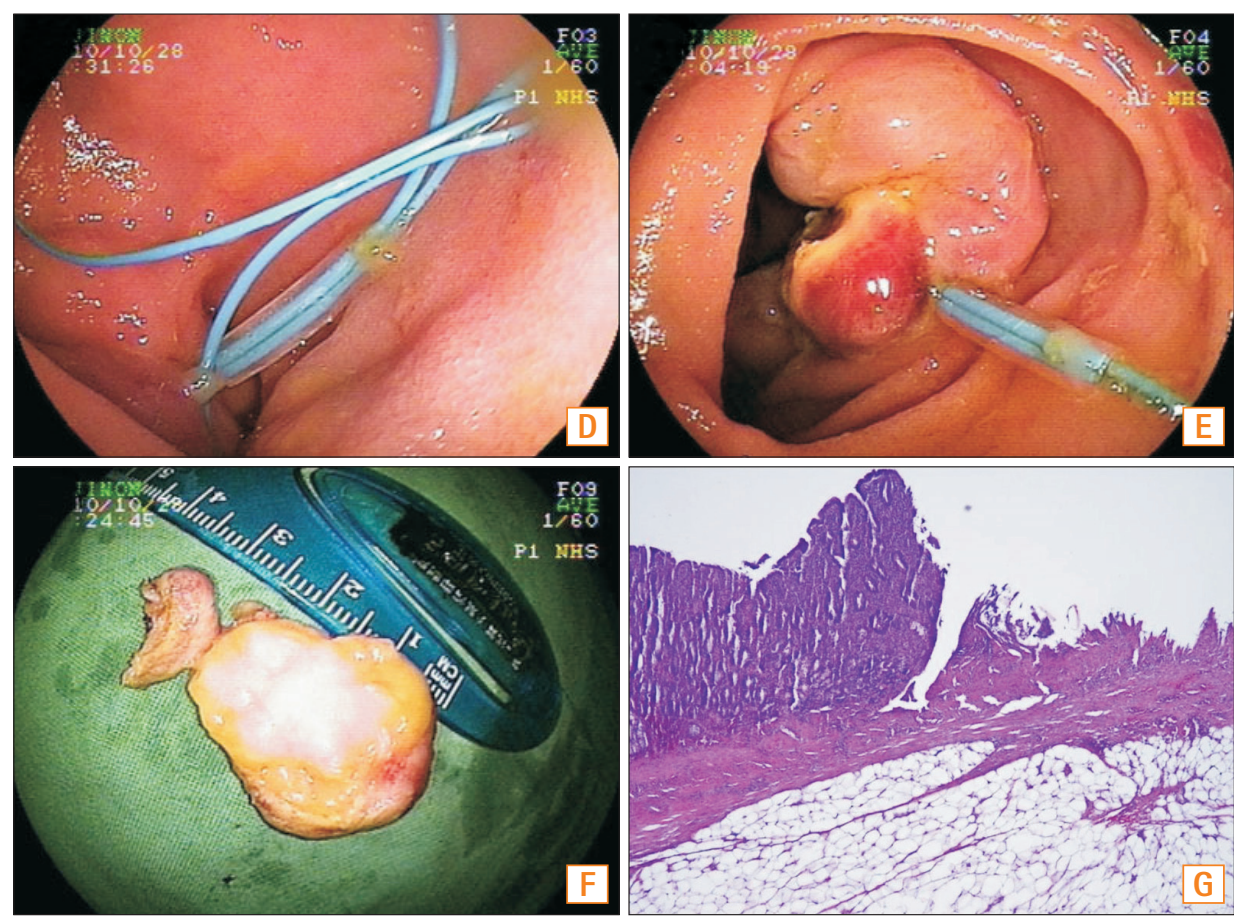

mor, a subtotal polypectomy using an electrosurgical snare was performed (Fig. E). The tumor showed a positive "naked fat sign" on the cutting surface after polypectomy (Fig. F). Pathological evaluation of the resected tumor revealed much adipose tissue with superficial ulceration (Fig. G. H\&E, $\times 40$ ). What is your diagnosis of this subepithelial tumor?

\section{Answer to the Images: Jejunal Lipoma}

Small bowel lipomas are rare and often asymptomatic. If symptomatic, they present with abdominal pain, hemorrhage, intestinal obstruction, intussusception, or perforation. ${ }^{1}$ Lipomas are more common in the ileum than in the duodenum and jejunum. ${ }^{1}$ These tumors are difficult to diagnose preoperatively. On endoscopic examination, lipomas are characteristically smooth, yellowish, round, or hemispherical tumors with either a pedunculated or wide base. The "cushion sign" and "naked fat sign" are endoscopic characteristics of lipomas. Balloon-assisted enteroscopy is a powerful useful modality for detecting small bowel lipomas. ${ }^{2}$ Surgical or endoscopic resection is needed for most symptomatic lipomas; however, it is not clear whether asymptomatic lipomas require any intervention. ${ }^{3}$ Endoscopic resection of lipomas is a challenge for endoscopists because fatty tissue of lipoma is an inefficient conductor of electric current and results in an increased risk of hemorrhage or perforation. Endoloopassisted polypectomy for lipomas have been reported previously, mostly in the colon and duodenum. ${ }^{4,5}$ Herein, we report for the first time a case of a large symptomatic jejunal lipoma treated with endoloop-assisted polypectomy using spiral enteroscopy.

\section{REFERENCES}

1. Wilson JM, Melvin DB, Gray G, Thorbjarnarson B. Benign small bowel tumor. Ann Surg 1975;181:247-250.

2. Chou JW, Feng CL, Lai HC, et al. Obscure gastrointestinal bleeding caused by small bowel lipoma. Intern Med 2008;47:16011603.

3. Yu HG, Ding YM, Tan S, Luo HS, Yu JP. A safe and efficient strategy for endoscopic resection of large, gastrointestinal lipoma. Surg Endosc 2007;21:265-269.

4. Koo J, Kaffes A. Endoscopic resection of large colonic lipomas assisted by a prototype single-use Endoloop device. Endoscopy 2006;38:644-647.

5. Huang WH, Peng CY, Yu CJ, Chou JW, Feng CL. Endoloopassisted unroofing for the treatment of symptomatic duodenal lipomas. Gastrointest Endosc 2008;68:1234-1236. 